KIMIKA Volume 31, Number 2, pp. 1-4 (2019)

(C) 2019 Kapisanang Kimika ng Pilipinas

All rights reserved. Published in the Philippines.

ISSN 0115-2130 (Print); 2508-0911 (Online)

https://doi.org/10.26534/kimika.v30i2.1-4

\title{
CHARACTERISTICS AND KINETICS OF PHOSPHORUS RELEASE FROM FORMULATED FERTILIZER IN GLAY LOAM SOIL
}

\author{
Vernadette J. Soriano, Veronica P. Migo, Monet Concepcion Maguyon-Detras, Catalino G. \\ Alfafara \\ Department of Chemical Engineering, College of Engineering and Agro-Industrial Technology, \\ University of the Philippines, Los Baños, Laguna, Philippines 4031
}

\begin{abstract}
A kinetic study for the phosphorus release of a formulated CaHAP-Z fertilizer was done to observe its behavior when applied to clay loam soil. The study of release kinetics of CaHAP-Z was done along with CaHAP and the control fertilizer Solophos ${ }^{\mathrm{TM}}$ to determine whether the formulated fertilizer can be an alternative for the conventional fertilizer. Results showed that the formulated fertilizer CAHAP-Z contains 3.73\% phosphorus with $513.10 \mathrm{~nm}$ particle (Dynamic Light Scattering) indicating that the formulated fertilizer can be classified as a nanofertilizer. The formulated CaHAP$\mathrm{Z}$ fertilizer showed the slowest release kinetics compared to the controls. In addition, this study showed that the simple Elovich kinetic model is the general equation that best fits to describe the phosphorus release of fertilizers.
\end{abstract}

Keywords: Nanofertilizer; Phosphorus; Kinetics; Release Behavior; Clay Loam Soil; Zeolite

\section{INTRODUCTION}

Fertilizers have been considered as a solution to food shortage due to its promising effect on increasing the crop yield. In developing an effective fertilizer, it is important to know the elements that are essential to support plant growth. One of the macronutrients needed by the plants is phosphorus (McKenzie, 1998; Solanki et al., 2015). Insufficient supply of phosphorus would cause stunted growth for the plants (Solanki et al. 2015). On the other hand, too much supply causes the excess to be washed out into bodies of water, which results to eutrophication damaging the water ecosystem by enhancing the growth of algae (Busman et al., 2002).

In order to maximize the nutrient uptake of plants and to minimize the environmental damages, nanotechnology is applied to formulate fertilizers since nutrients are more efficiently absorbed by plant pores when nutrients are in nanoscale. Improved absorption would also lead to minimized loss of the fertilizer. This study mainly aims to

\footnotetext{
*Author to whom correspondence should be addressed; email: vjsoriano1@up.edu.ph
} 
evaluate the release pattern of a phosphorus nanofertilizer through its release kinetics in clay loam soil, one of the most common soils in the Philippines.

\section{EXPERIMENTAL}

Experimental Set Up. CaHAP (calcium hydroxyphosphate, $\mathrm{HCa}_{5} \mathrm{O}_{13} \mathrm{P}_{3}$ ) was obtained from Carl Roth Gmbh Co. \& Kg. The zeolite (clinoptilolite) and the control Solophos ${ }^{\mathrm{TM}}$ were obtained from Saile Industries. The CaHAP-Z was synthesized using the method described by Subbaiya et al (2012). Four (4) soil columns were used, each with their own mariotte bottles, one for each of the treatments: blank, CaHAP, CaHAP-Z, and Solophos $^{\mathrm{TM}}$. The columns were filled with clay loam soil up to a height of $20 \mathrm{~cm}$ (approximately 350g of clay loam soil). Fertilizer loading was done with the adapted 1:100 ratio of fertilizer to soil weight, therefore each column had 3.5g of fertilizer applied. Water was applied and maintained with a $3 \mathrm{~cm}$ height over the soil, and was continuously replenished every leachate collection which was done every 24 hours for 30 days.

Experimental Procedures. Collection of leachate was done by opening the valve and collecting a fair amount of leachate, approximately until the $3 \mathrm{~cm}$ water height was exhausted, then closing the valve and replenishing the water lost. To determine the total phosphorus content of the various phosphorus fertilizers that were applied to the soil, phosphovanado-molybdate complex was used for the colorimetric analysis of the samples for the study of their release kinetics. The phosphovanado-molybdate complex was synthesized by dissolving $1.25 \mathrm{~g}$ of ammonium vanadate in $500 \mathrm{~mL}$ of $5 \mathrm{~N} \mathrm{HNO}_{3}$, mixed with $25 \mathrm{~g}$ of ammonium molybdate dissolved in $300 \mathrm{~mL}$ distilled $\mathrm{H}_{2} \mathrm{O}$. The final product was then diluted to $1 \mathrm{~L}$.

The determination of the total phosphorus concentration of the leachate samples were determined by using the equation:
$\% \mathrm{P}=\frac{\sum[(\mathrm{P} \text { conc. of leachate }) \times \mathrm{DF} \times(\text { vol. of leachate })]}{(\text { mass } \mathrm{P} \text { of fertilizer applied })} \times 100$

where DF is the dilution factor, $\frac{\text { final volume of solution }}{\text { volume of sample used }}=\frac{25}{1} \quad$ and the final volume of solution refers to the volume after addition of mixed reagent and diluting to mark.

\section{RESULTS AND DISCUSSION}

The results of the SEM analysis of CaHAP-Z showed the particles with sizes between 42.0 - $63.0 \mathrm{~nm}$, which are all under $100 \mathrm{~nm}$ and are classified as nanoparticles. Analysis using Dynamic Light Scattering (DLS) however, revealed the particle size of CaHAP-Z averaging at $513.10 \mathrm{~nm}$. The difference in particle size observed in SEM and DLS is attributed to the presence of water molecules on the surface of the nanoparticles. Assuming that a hydration layer is surrounding the particle, the measured diameter from the particle's diffusional properties indicates the size of the solvated particle, which is called the hydrodynamic diameter instead of the core diameter of the particle (Rosbero and Camacho, 2017). Based on the generally accepted definition of nanoparticles, a fertilizer particle of $513 \mathrm{~nm}$ hydrodynamic size might not qualify as a nanofertilizer, however in agricultural perspective, particles up to $1000 \mathrm{~nm}$ size can be classified as nanoparticle (Mukhopadhyay 2014).

Colorimetric analysis using phosphovanadomolybdate complex showed the CaHAP-Z fertilizer to have $3.37 \%$ phosphorus content, while CaHAP and Solophos ${ }^{\mathrm{TM}}$ have $13.14 \%$ and $10.52 \%$ respectively. This method has a detection range of $1-20 \mathrm{mg} \mathrm{P} / \mathrm{L}$ and uses the formation of molybdophosphoric acid from ammonium molybdate placed under acidic conditions. Yellow phosphovanadomolybdate complex is formed when vanadium is added, and the intensity of the color indicates phosphate concentration (Kitson \& Mellon, 1994). Figure 1 shows the percent phosphorus release versus time 
graph for the duration of the thirty-day observation period.

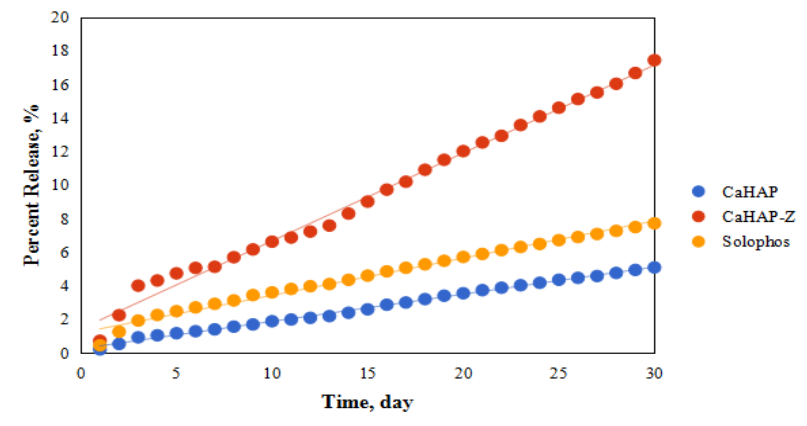

Figure 1. Percent phosphorus release versus time of CaHAP, CaHAP-Z and Solophos ${ }^{\mathrm{TM}}$.

The "blank" was used as a correction to account for the phosphorus in the soil. Its $\mathrm{P}$ release was subtracted from the $P$ release of the fertilizers tested. The fertilizers displayed an almost linear trend, with the exception of the first few points of the CaHAP-Z fertilizer. The first three points of CaHAP-Z, which indicates the first three days of leaching, has larger percent release, before it fell along the linear trend.

There are three possible models to fit the release kinetics of the fertilizers. These are the first order, second order, and simple Elovich model. Kinetics of first order and second order occur when the ratio of the phosphorus release and the concentration of phosphorus remaining in the fertilizer is constant at any given time. A fertilizer of second order is more likely to have faster release kinetics as compared to a fertilizer following first order at near equal rate constant. According to Shariatmadari et al. (2006), the rate of phosphorus release can be fitted to multiple first-order equations that will describe the different stages of phosphorus release. Compared to fitting the release data to two or three first order equations, the simple Elovich equation can be successfully used to describe the release kinetics using only a single line (Chien and Clayton, 1980). All fertilizers were fitted into the first order, second order and simple Elovich model.

The model which best fits the data from the soil column is the Simple Elovich model. Compared to fitting the release data to two or three first order equations, the simple Elovich equation can be successfully used to describe the release kinetics using only a single line (Chien and Clayton, 1980). The Simple Elovich model takes the concentration of the phosphorus (dependent variable) in terms of parts per million and plotting it against the natural logarithm of time (independent variable), which in this case was expressed in terms of day. Based from the definition of Shariatmadari et al. (2006), where a high rate value $(\alpha)$ and a low constant value $(\beta)$ signifies faster release kinetics, Solophos ${ }^{\mathrm{TM}}$ would have the fastest release kinetics among the three fertilizers analyzed. It has both the fastest rate and lowest constant value. This is followed by the CaHAP, then CaHAP-Z fertilizer for the slowest release kinetics. The rate kinetics suggests the possibility of the formulated fertilizer as a slow release fertilizer for the delivery of an important plant nutrient. Detailed investigations on the slow release of CaHAP-Z are underway and will be reported elsewhere.

Table 1. Goodness of fit to the model equations and rate constants of Simple Elovich model of each fertilizer.

\begin{tabular}{llllll}
\hline \multirow{2}{*}{ FERTILIZER } & \multicolumn{2}{l}{ COEFFICIENTS OF DETERMINATION } & \multicolumn{2}{l}{ RATE CONSTANTS } \\
\cline { 2 - 6 } & 1st-order & 2nd-order & Simple Elovich & B (ppm-1) & 目 (ppm/day) \\
\hline CaHAP & 0.8322 & 0.3602 & 0.893 & 0.0122 & 44.2550 \\
CaHAP-Z & 0.8017 & 0.3152 & 0.8917 & 0.0135 & 43.9530 \\
SolophosTM & 0.7701 & 0.3394 & 0.9455 & 0.0111 & 63.3246 \\
\hline
\end{tabular}




\section{CONCLUSIONS}

In conclusion, CaHAP-Z fertilizer fits the simple Elovich model equation to describe phosphorus release. As such, the release kinetics of the fertilizer must be explored and the variables that affect the release rate of phosphorus should be further investigated.

\section{REFERENCES}

Busman L, Lamb J, Randall G, Rehm G, Schmitt M. Nutrient Management: The nature of phosphorus in soils. Regents of the University of Minnesota, 2002.

http://www.extension.umn.edu/distribution /cropsystems/dc695.html

Chien SH, Clayton WR. Application of Elovich equation to the kinetics of phosphate release and sorption in soils. Soil Sci Soc Am J. 1980 Mar; 44(2):265-268. https://doi.org/10.2136/sssaj1980.0361599 5004400020013x

Kitson RE, Mellon MG. Colorimetric determination of phosphorus as molybdivanadophosphoric acid. Ind Eng Chem Anal Ed. 1944 Jun; 16(6):379-383. https://doi.org/10.1021/i560130a017

Mukhopadhyay SS. Nanotechnology in agriculture: prospects and constraints. Invited Review. Nanotechnol Sci Appl. 2014 Aug; 4(7):63-71. doi: 10.2147/NSA.S39409\

Mckenzie R. Crop nutrition and fertilizer requirements. Lethbridge:Alberta Agriculture Food and Rural Development; 1998.

Rosbero TMS, Camacho DH. Green preparation and characterization of tentaclelike silver/copper nanoparticles for catalytic degradation of toxic chlorpyrifos in water. J Environ Chem Eng. 2017 Jun; 5(3):25242532.

https://doi.org/10.1016/j.jece.2017.05.009
Shariatmadari H, Shirvani M, Jafari A. Phosphorus release kinetics and availability in calcareous soils of selected arid and semiarid topo sequences. Geoderma. 2006 Jun; 132(3-4):261-272. https://doi.org/10.1016/j.geoderma.2005.05 .011

Solanki P. Bhargava A. Chhipa H, Jain N, Panwar J. Nano-fertilizers and their smart delivery system. In: Rai M, Ribeiro C, Mattoso L, Duran N. editors. Nanotechnologies in Food and Agriculture. Switzerland: Springer International Publishing; 2015. p. 81-102.

Subbaiya R, Priyanka M, Masilamani Selvam $M$. Formulation of green nano-fertilizer to enhance the plant growth through slow and sustained release of nitrogen. J Pharm Res. $2012 \quad$ Nov; 5(11):5178-5183. 